\title{
Stellar filaments in self-interacting Brans-Dicke gravity
}

\author{
M. Sharif ${ }^{1, a}$, Rubab Manzoor ${ }^{1,2, b}$ \\ ${ }^{1}$ Department of Mathematics, University of the Punjab, Quaid-e-Azam Campus, Lahore 54590, Pakistan \\ 2 Department of Mathematics, University of Management and Technology, Johar Town Campus, Lahore 54782, Pakistan
}

Received: 23 January 2016 / Accepted: 6 April 2016 / Published online: 18 May 2016

(C) The Author(s) 2016. This article is published with open access at Springerlink.com

\begin{abstract}
This paper is devoted to the study of the cylindrically symmetric stellar filaments in self-interacting BransDicke gravity. For this purpose, we construct polytropic filamentary models through a generalized Lane-Emden equation in the Newtonian regime. The resulting models depend upon the values of the cosmological constant (due to the scalar field) along with the polytropic index and represent a generalization of the corresponding models in general relativity. We also investigate the fragmentation of the filaments by exploring the radial oscillations through a stability analysis. This stability criterion depends only upon the adiabatic index.
\end{abstract}

\section{Introduction}

Filamentary structures have important implications in structure formation of the universe. These stellar configurations are omnipresent in the universe at various scales. On cosmological scales, the cosmic filament is associated with the cosmic web [1-4] where it behaves like a bridge connecting various dense regions (galaxies). On small scales, filaments are the features of the interstellar medium and instabilities within these filaments form a dense medium which turns into a star [5]. The stellar filamentary structures appear in a variety of astronomical contexts; their dynamical analysis can be considered as an effective tool to understand the behavior of galactic as well as interstellar structures in the universe.

It happens very often that the geometries of the proposed models are taken to be very simple or ideal, partly because of reasons like mathematical simplicity which may avoid complexities in the analysis, to obtain some results of physical interest, and to provide an ideal model which can act as a foundation and can be modified according to the required astrophysical as well as cosmological applications. The cylindrically symmetric configuration is one of the ideal

\footnotetext{
a e-mail: msharif.math@pu.edu.pk

be-mail: rubab.manzoor@umt.edu.pk
}

cases, which has widely been used to represent filamentary structures in the universe. In 1953, Chandrasekhar and Fermi [6] described the dynamics of a cylinder filled with a homogeneous and incompressible fluid. Stodolkiewicz [7] developed the magneto-hydrodynamics equilibrium of isothermal cylindrical filaments. Ostriker [8,9] studied the cylindrically symmetric filamentary structure with a polytropic equation of state and generalized the dynamical analysis of homogeneous compressible filaments. Afterward, many researchers explored cylindrical filamentary structures both analytically as well as numerically [10-14]. Recently, Breysse et al. [15] described polytropic filamentary structures through a stability analysis of the cylindrically symmetric self-gravitating fluid.

Modified theories of gravity constructed by modifying the Einstein-Hilbert action are considered to be candidates for the accelerating agent (dark energy) of the expanding universe [16-19]. There is a large body of literature [20-25] which has explored the dynamics of stellar structures in modified gravity to reveal the modification hidden in the structure formation of the universe. Brans-Dicke (BD) gravity, being a natural generalization of general relativity (GR) $[26,27]$, is one of the fascinating examples of modified gravity, which is considered as a solution of various cosmic problems. One of the main features of this theory is that the gravitational force is described by a massless scalar field $\phi$ along with a curvature part (Ricci scalar). It also contains a coupling constant $\omega_{\mathrm{BD}}$ which serves as a tunable parameter to adjust the required results. This theory provides suitable solutions of many cosmic issues but remains unable to describe the "graceful exist" problem of old inflationary cosmology. The inflationary model of this theory is valid for a specific value of the coupling parameter $\omega_{\mathrm{BD}} \leq 25$ [28] which contradicts observational data [29]. Moreover, the observational limit of $\omega_{\mathrm{BD}}$ at small scale (weak field) [30,31] is inconsistent with those calculated at large scale [32].

These issues were resolved in self-interacting BD (SBD) gravity, which is developed by introducing a massive poten- 
tial function $V(\phi)$ in the Jordan framework of BD gravity [33]. The potential function represents a massive scalar field term which describes the potential of the scalar field $(\phi)$ or the potential of the energy density related to the scalar field in the system. This theory provides consistency between the weak field and the strong field regimes [34]. Recent literature indicates the dynamics of SBD gravity in many cosmic problems [35-40]. In recent papers [41-43], we have explored spherically as well as cylindrically symmetric selfgravitating fluids in SBD gravity and found some interesting results. We have also investigated hydrodynamics and oscillations of spherically symmetric gaseous distributions in the post-Newtonian approximations of this theory $[44,45]$.

In this paper, we study filamentary structures of stellar systems in SBD theory. We construct cylindrical polytropic models of the filaments in the Newtonian $(\mathrm{N})$ approximation and explore the fragmentation of filamentary structures through radial oscillations of self-gravitating fluids. The paper is organized as follows. Section 2 formulates SBD as well as dynamical equations in the $\mathrm{N}$ limit. In Sect. 3 , we construct cylindrical polytropic filamentary models in $\mathrm{N}$ approximation. Section 4 is devoted to a discussion of radial oscillations of the filaments through a stability analysis. Finally, Sect. 5 summarizes the results.

\section{Self-interacting Brans-Dicke gravity and dynamical equations}

The action of SBD gravity [33] is given by

$$
\begin{aligned}
S= & \frac{1}{2 \kappa^{2}} \int d^{4} x \sqrt{-g}\left[\phi R-\frac{\omega_{\mathrm{BD}}}{\phi} \nabla^{\alpha} \phi \nabla_{\alpha} \phi-V(\phi)\right] \\
& +L_{m},
\end{aligned}
$$

where $\kappa^{2}=\frac{8 \pi G}{c^{2}}$ and $L_{m}$ shows the matter contribution. The variation of this action with respect to the scalar field $(\phi)$ and the metric tensor $\left(g_{\mu \nu}\right)$ provides the SBD equations as follows:

$$
\begin{aligned}
G_{\mu \nu}= & \frac{\kappa^{2}}{\phi} T_{\mu \nu}+\frac{1}{\phi}\left[\phi_{, \mu ; \nu}-g_{\mu \nu} \square \phi\right] \\
& +\frac{\omega_{\mathrm{BD}}}{\phi^{2}}\left[\phi_{, \mu} \phi_{, \nu}-\frac{1}{2} g_{\mu \nu} \phi_{, \alpha} \phi^{, \alpha}\right]-\frac{V(\phi)}{2 \phi} g_{\mu \nu}, \\
\square \phi= & \frac{\kappa^{2} T}{3+2 \omega_{\mathrm{BD}}}+\frac{1}{3+2 \omega_{\mathrm{BD}}}\left[\phi \frac{\mathrm{d} V(\phi)}{\mathrm{d} \phi}-2 V(\phi)\right],
\end{aligned}
$$

where $T_{\mu \nu}$ represents the energy-momentum tensor of matter, $T=g^{\mu \nu} T_{\mu \nu}$, and $\square$ stands for the d'Alembertian operator. Equations (2) and (3) provide the field equations and evolution of the scalar field, respectively. We assume a matter contribution in the form of a perfect fluid, which can be compatible with the $\mathrm{N}$ regime,

$T_{\mu \nu}=\left(\rho c^{2}+p\right) u_{\mu} u_{\nu}-p g_{\mu \nu}$, where $\rho, p, u_{\mu}$ stand for density, pressure, and four velocity, respectively.

\subsection{Newtonian approximation}

The weak field approximated solutions of any relativistic theory describe the order of deviations or perturbation of the local system from its homogeneous and isotropic vacuum background. The $\mathrm{N}$ and parameterized post-Newtonian limits are widely used weak field approximated solutions that are derived by a Taylor expansion of the metric functions as follows:

$g_{\mu \nu} \approx \eta_{\mu \nu}+h_{\mu \nu}$

with

$h_{00} \approx h_{00}^{(2)}+h_{00}^{(4)}+\cdots$,

$h_{0 i} \approx h_{0 i}^{(3)}+h_{0 i}^{(5)}+\cdots$,

$h_{i j} \approx h_{i j}^{(2)}+h_{i j}^{(4)} \ldots$.

Here $\eta_{\mu \nu}$ indicates the Minkowski metric (describing a homogeneous and isotropic vacuum background of $\left.g_{\mu \nu}\right), h_{\mu \nu}$ shows the deviation of $g_{\mu \nu}$ from its background values $\left(\eta_{\mu \nu}\right)$, $i, j=1,2,3$ and the superscripts (2), (3), and (4) represent the order of approximation $\left(c^{-2}\right),\left(c^{-3}\right)$ as well as $\left(c^{-4}\right)$. The $\mathrm{N}$ limits require the information of $g_{00} \sim \eta_{00}+h_{00}^{(2)}, g_{i j} \sim$ $\eta_{i j}$, whereas the parameterized post-Newtonian corrections use the approximations $g_{00} \sim \eta_{00}+h_{00}^{(2)}+h_{00}^{(4)}, g_{0 i} \sim h_{o i}^{(3)}$, and $g_{i j} \sim \eta_{i j}+h_{i j}^{(2)}$. Thus, the $\mathrm{N}$ limits of any system can be directly obtained from its known parameterized postNewtonian approximations.

In order to discuss polytropic geometry in SBD gravity and check the compatibility of our results with the analysis of GR [15], we approximate the system in the $\mathrm{N}$ limits. For this purpose, we evaluate the $\mathrm{N}$ limits of SBD gravity from its known post-Newtonian approximate solutions. The parameterized post-Newtonian approximation of SBD (massive BD gravity) solutions has been evaluated by using the following Taylor expansion of the metric and the dynamical scalar field [46]:

$g_{\mu \nu} \approx \eta_{\mu \nu}+h_{\mu \nu}$

$\phi \approx \phi_{0}\left(t_{0}\right)+\varphi^{(2)}(t, x)+\varphi^{(4)}(t, x)$,

$V(\phi) \approx V_{0}+\varphi V_{0}^{\prime}+\varphi^{2} V_{0}^{\prime \prime} / 2+\cdots$

Here $\phi_{0}$ describes the dynamical scalar field as a function of background cosmic time $t_{0}$ (which varies slowly with respect to cosmic time $\left.t_{0}\right), V_{0}=V\left(\phi_{0}\right)$ is the potential function of the scalar field at $t_{0}$, and $\varphi(t, x)$ represents local deviation of the scalar field from $\phi_{0}$. The lowest-order parameterized post-Newtonian corrections $\left(O\left(c^{-2}\right)\right)$ of the SBD solutions are given by 


$$
\begin{aligned}
& g_{00} \approx 1-h_{00}^{(2)}=1-\frac{2 U}{c^{2}}+\frac{V_{0} r^{2}}{6 \phi_{0} c^{2}}, \\
& g_{i j} \approx-\left[1+h_{i j}^{(2)}\right] \delta_{i j}=\left[-1-\frac{2 \gamma_{\mathrm{BD}} U}{c^{2}}-\frac{V_{0} r^{2}}{6 \phi_{0} c^{2}}\right] \delta_{i j}, \\
& \frac{\varphi^{(2)}}{\phi_{0}} \approx \frac{-2 U}{c^{2}}\left[\frac{e^{-m_{0} r}}{3+2 \omega_{\mathrm{BD}}+e^{-m_{0} r}}\right] .
\end{aligned}
$$

Here $U=G_{\text {eff }} \frac{M_{\odot}}{r}\left(M_{\odot}\right.$ is the Newtonian mass of the sun) shows the effective gravitational potential determined by the Poisson equation

$\nabla^{2} U=-4 \Pi \rho G_{\mathrm{eff}}$,

where

$$
\begin{aligned}
G_{\mathrm{eff}} & =\frac{\kappa^{2}}{8 \pi \phi_{0}}\left(1+\frac{e^{-m_{0} r}}{3+2 \omega_{\mathrm{BD}}}\right), \\
m_{0} & =\left(\frac{\phi_{0} V_{0}^{\prime \prime}-V_{0}^{\prime}}{3+2 \omega_{\mathrm{BD}}}\right)^{1 / 2} .
\end{aligned}
$$

The term $\gamma_{\mathrm{BD}}$ represents the parameterized post-Newtonian parameter given by

$\gamma_{\mathrm{BD}}=\frac{3+2 \omega_{\mathrm{BD}}-e^{-m_{0} r}}{3+2 \omega_{\mathrm{BD}}+e^{-m_{0} r}}$,

where $m_{0}$ represents mass of the massive scalar field with constraint $\left(m_{0}>>\frac{1}{\tilde{r}}\right)$ (where $\tilde{r}$ represents the scale of the experiment or observation testing the field). When the background value of this mass is very small $\left(m_{0}<<\frac{1}{\tilde{r}}\right)$ the SBD system reduces to simple BD gravity (the massive scalar field reduces to a massless scalar field) having the post-Newtonian parameter

$\gamma_{\mathrm{BD}}=\frac{1+\omega_{\mathrm{BD}}}{2+\omega_{\mathrm{BD}}}$.

That is why the BD theory (massless scalar field) is consistent with solar system constraints of the Cassini mission for $\omega_{\mathrm{BD}}>40,000$. However, for massive BD gravity (SBD gravity), the dynamics of the spatial part of $\phi$ is frozen on the solar system scales through the potential function and all values of $\omega_{\mathrm{BD}}$ are observationally acceptable [47-49].

The term $\frac{V_{0} r^{2}}{6 \phi_{0} c^{2}}=\frac{\Lambda_{\mathrm{BD}} r^{2}}{3 c^{2}}$ describes the cosmological constant term which depends upon the potential of the scalar field. In order to be consistent with observational data ranging from the solar system to clusters of galaxies the contribution due to the scalar density should be very small and the following constraint must be satisfied:

$\frac{V_{0} L^{2}}{\phi_{0}}<<1$.

Here $L$ indicates the length scale equal to or greater than the solar system.

The $\mathrm{N}$ limits of any scalar-tensor theory require information of $g_{00} \sim \eta_{00}+h_{00}^{(2)}, g_{i j} \sim \delta_{i j}$ and $\phi \sim \phi_{0}$. Thus, from the parameterized post-Newtonian analysis of SBD theory the obtained $\mathrm{N}$ approximated SBD solutions are evaluated as

$g_{00} \approx 1-h_{00}^{(2)}=1-\frac{2 U}{c^{2}}+\frac{\Lambda_{\mathrm{BD}} r^{2}}{3 c^{2}}$,

$g_{i j} \approx-\delta_{i j}, \quad \phi \approx \phi_{0}$.

From now on, we use $h_{00}^{(2)}=h_{00}$ for the sake of convenience. It may be noticed that, by applying the limits $\left(m_{0}<<\frac{1}{\tilde{r}}\right)$ and $\frac{V_{0}}{\phi_{0}} \rightarrow 0$, the above defined $\mathrm{N}$ approximations of SBD gravity can be shifted to $\mathrm{N}$ limits of BD solutions. Similarly, in the limits $\left(m_{0}<<\frac{1}{\tilde{r}}\right), \frac{V_{0}}{\phi_{0}} \rightarrow 0$ and $\omega_{\mathrm{BD}} \rightarrow \infty$, the obtained approximated system can be converted into the GR case.

\subsection{Dynamical equations}

The polytropic geometry of any configuration is based upon two dynamical equations, the Poisson equation and the equation of motion of the respective system [15]. Here, we calculate the generalized form of both dynamical equations for SBD gravity. Equation (2) can be rewritten as

$$
\begin{aligned}
R_{\mu \nu}= & \frac{\kappa^{2}}{\phi}\left(T_{\mu \nu}-\frac{1}{2} g_{\mu \nu} T\right)+\frac{\omega_{B D}}{\phi^{2}}\left[\phi_{, \mu} \phi_{, \nu}\right] \\
& +\frac{1}{\phi}\left[\phi_{, \mu ; \nu}\right]+\frac{g_{\mu \nu}}{2 \phi}[\square \phi+V(\phi)] .
\end{aligned}
$$

The temporal component of this equation in $\mathrm{N}$ approximation is given by

$R_{00}=\frac{1}{2}\left(\frac{k^{2} \rho}{\phi_{0}}-\frac{V_{0}}{\phi_{0}}\right)$,

where the contributions due to $\dot{\phi}_{0}$ and $\ddot{\phi}_{0}$ are neglected because the term $\phi_{0}$ behaves as almost a constant. The expansion of Ricci tensor in the $\mathrm{N}$ regime is approximated by

$R_{00}=-\frac{1}{2} \nabla^{2} h_{00}$

Comparing these two values, we obtain the generalized Poisson equation as follows:

$\nabla^{2} h_{00}=-\frac{k^{2} \rho}{\phi_{0}}+\frac{V_{0}}{\phi_{0}}$,

where $h_{00}$ represents the gravitational potential due to matter as well as the massive scalar field. The generalized Euler equations can be obtained by using $T_{; v}^{\mu \nu}=0$, whose time component yields the continuity equation,

$\frac{\partial \rho}{\partial t}+\frac{\partial}{\partial x_{i}}\left(\rho v_{i}\right)=0$

where $v_{i}(i=1,2,3)$ stands for the components of the velocity. The spatial components give the generalized form of the equation of motion as follows:

$\rho \frac{\partial v_{i}}{\partial t}=-\frac{\partial p}{\partial x_{i}}-\left(\rho c^{2}+p\right) \frac{\partial\left(\ln g_{00}\right)}{\partial x_{i}}$. 


\section{Cylindrical polytropes}

In order to discuss cylindrical polytropic filaments in SBD gravity, we consider the equilibrium configuration and the standard polytropic equation of state:

$p=K \rho^{\gamma}$,

where $K$ is a constant and $\gamma=\frac{n+1}{n}$ represents the polytropic exponent with $n$ as a polytropic index [15,50]. In hydrostatic equilibrium, the cylindrical configurations of Eqs. (12) and (14) turn out to be

$\frac{1}{r} \frac{\mathrm{d}}{\mathrm{d} r}\left(r \frac{\mathrm{d} h_{00}}{\mathrm{~d} r}\right)=-\frac{k^{2} \rho}{\phi_{0}}+\frac{V_{0}}{\phi_{0}}$,

$\frac{\mathrm{d} p}{\mathrm{~d} r}=-\left(\rho c^{2}+p\right) \frac{\mathrm{d}\left(\ln g_{00}\right)}{\mathrm{d} r}$.

Integration of Eq. (17) by using (15) provides

$\rho=\left[\frac{1}{K}\left(g_{00}^{-\frac{1}{2(n+1)}}-1\right)\right]^{n}$.

Using a binomial expansion on $g_{00}^{-\frac{1}{2(n+1)}}$ and approximating up to $O\left(c^{-2}\right)$, we have

$\left(g_{00}^{-\frac{1}{2(n+1)}}-1\right) \approx \frac{-1}{2(n+1)} h_{00}$.

Using Eqs. (19) into (18), we obtain the relation between the density and potential configurations as follows:

$\rho=C_{n}\left(-h_{00}\right)^{n}, \quad C_{n}=[2 K(n+1)]^{-n}$.

Equations (16), (20), and the cosmological constant term provide a differential equation for a gravitational potential $h_{00}$ as follows:

$\frac{1}{r} \frac{\mathrm{d}}{\mathrm{d} r}\left(r \frac{\mathrm{d} h_{00}}{\mathrm{~d} r}\right)=-\frac{k^{2} C_{n}\left(-h_{00}\right)^{n}}{\phi_{0}}+2 \Lambda_{\mathrm{BD}}$.

The above equation can be converted to a polytropic equation (Lane-Emden equation) by considering the following dimensionless variables:

$\beta(s)=\frac{h_{00}}{h_{00(c)}}=\left(\frac{\rho}{\rho_{c}}\right)^{\frac{1}{n}}, \quad s=\frac{r}{b}, \quad b=\left[C_{n} h_{00(c)}^{n-1}\right]^{-\frac{1}{2}}$,

where $h_{00(c)}$ and $\rho_{c}$ represent the potential as well as the density at the center of the cylinder $(r=0)[15,50]$. With these assumptions, Eq. (21) turns out to be

$\frac{1}{s} \frac{\mathrm{d}}{\mathrm{d} s}[s \beta]=-\beta^{n}+A, \quad A=\frac{2 \Lambda_{B D}}{h_{00(c)}}$.

This is a modified form of the original Lane-Emden equation describing spherical polytropic model [50] and generalized form of the modified Lane-Emden equation that describes the cylindrically symmetric polytropic filament in GR [15]. Here $\beta(s)$ is the Lane-Emden function describing the equilibrium potential and $A$ is considered as a cosmological constant term due to the presence of the scalar field. This is a second order differential equation satisfying the following boundary conditions: at the center $(r=0)$ we have $s=0, h_{00} \approx h_{00(c)}, \rho \approx \rho_{c}, \beta \approx 1$ and $\left(\frac{\mathrm{d} \beta}{\mathrm{d} s}\right)_{s=0}=0$. The surface of the polytopic filament is represented by a specific value $s=S$ for which the density $\rho$ as well as the Lane-Emden function $(\beta)$ becomes zero. Equation (23) can be solved analytically for different values of $n$. Some possible analytical solutions are given as follows:

$\beta(s)=1+\frac{s^{4}}{4}+\frac{A s^{2}}{4}, \quad n=0$,

$\beta(s)=-A+J_{0}(s)+A J_{0}(s), \quad n=1$,

where $J_{0}(s)$ is a Bessel function of zeroth order. It is noted that the analytical results depend upon the values of $s$ as well as $A$. We can also solve Eq. (23) numerically for different values of $n$ and $A$. Some numerical solutions of the modified Lane-Emden equation for $n=0,1,3,5$ with $A=0, \pm 1 / 2, \pm 1, \pm 3$ are given in Figs. 1, 2, 3, 4, 5, 6, 7 , and 8 , respectively.

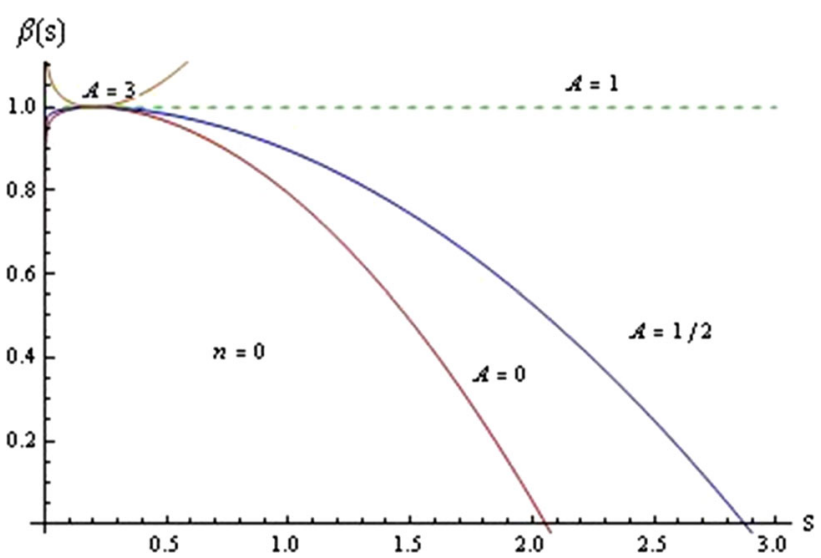

Fig. 1 Values of $\beta(s)$ for $n=0$ and $A=0,1 / 2,1,3$

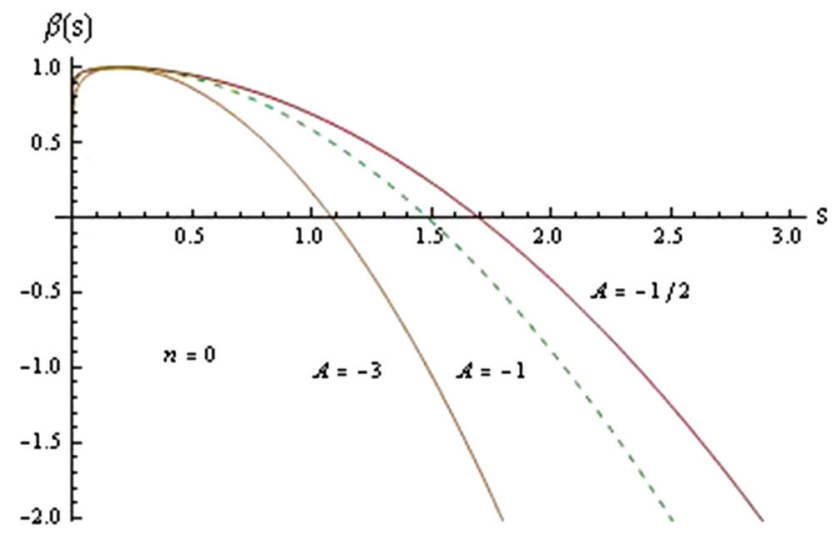

Fig. 2 Values of $\beta(s)$ for $n=0$ and $A=-1 / 2,-1,-3$ 

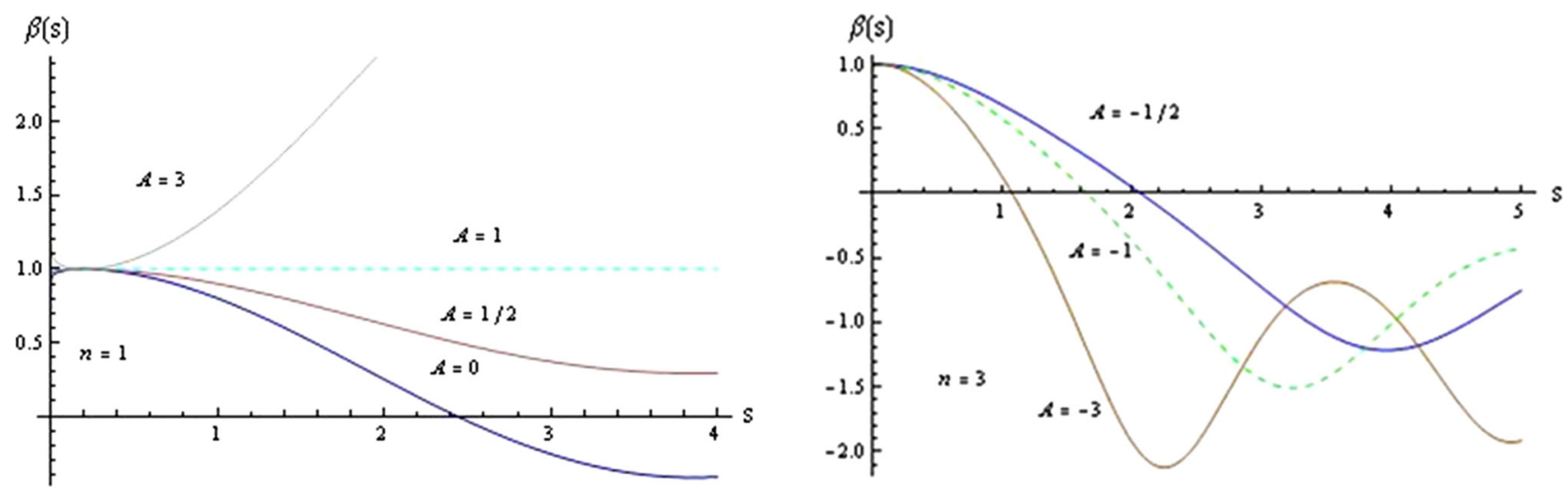

Fig. 3 Values of $\beta(s)$ for $n=1$ and $A=0,1 / 2,1,3$

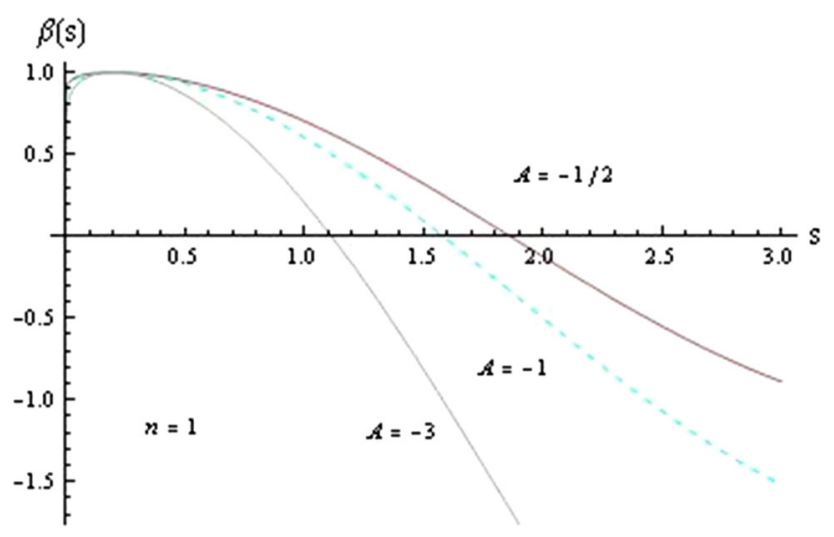

Fig. 6 Values of $\beta(s)$ for $n=3$ and $A=-1 / 2,-1,-3$

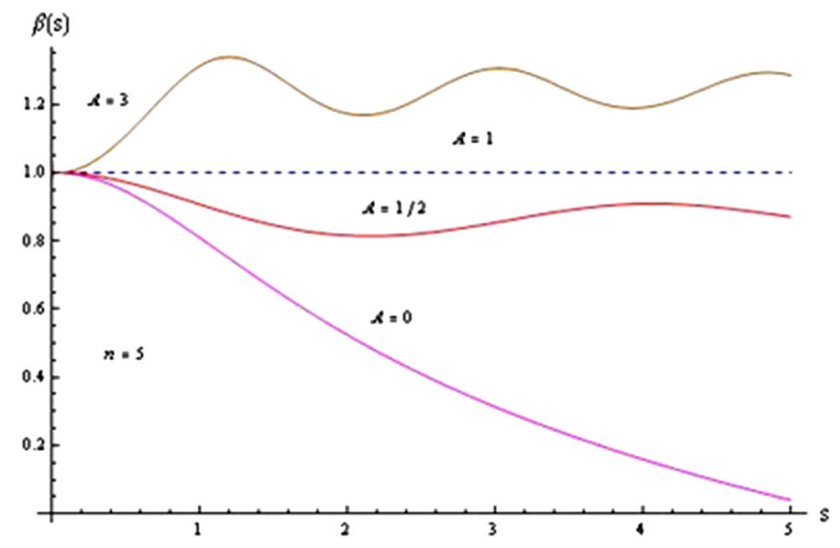

Fig. 4 Values of $\beta(s)$ for $n=1$ and $A=-1 / 2,-1,-3$

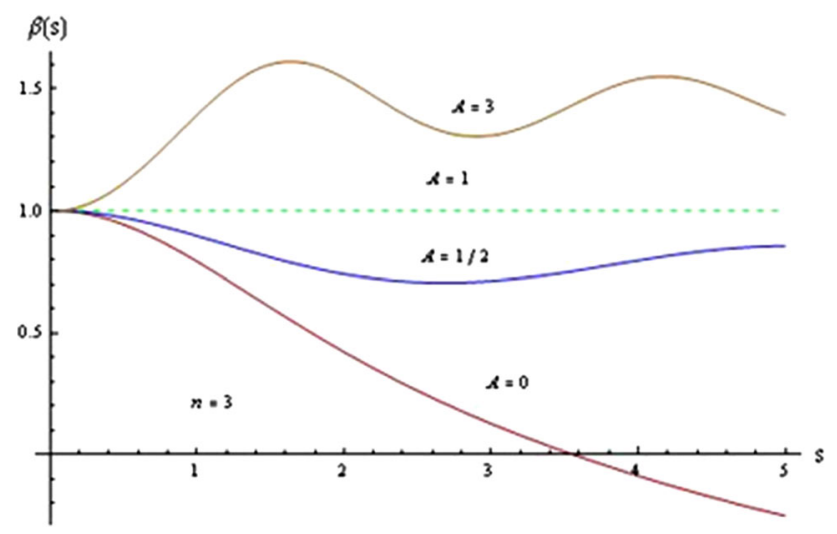

Fig. 7 Values of $\beta(s)$ for $n=5$ and $A=0,1 / 2,1,3$

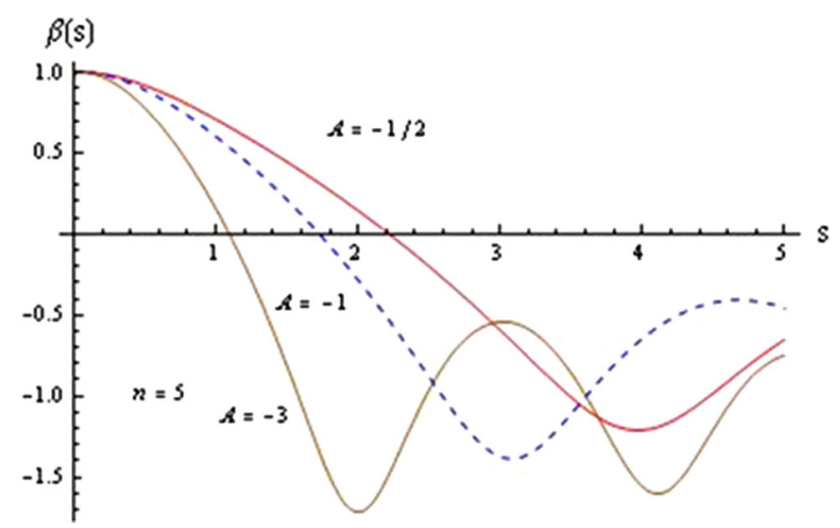

Fig. 5 Values of $\beta(s)$ for $n=3$ and $A=0,1 / 2,1,3$

Figures 1 and 2 show polytropic models for $n=0$ with $A=0, \pm 1 / 2, \pm 1, \pm 3$. It is found that the polytropic filaments for $A \lesssim 1 / 2$ have finite radii, i.e., after taking finite values of $s$, the polytropic function $\beta$ becomes zero. For $A>1 / 2$, the polytropic models have infinite radii. Figures 3 and 4 show that, for $n=1$, we have finite radii models for $A \lesssim 0$ and infinite radii models for $A>0$. Figures 5 and 6 represent polytropic filaments for $n=3$ where $A \lesssim 0$ represents finite radii, while $A>0$ gives infinite radii models.

Fig. 8 Values of $\beta(s)$ for $n=5$ and $A=-1 / 2,-1,-3$

Figures 7 and 8 show models for $n=5$ and the results are almost similar to the cases $n=1,3$.

It can be noticed that under the constraint $\left(m_{0}<<\frac{1}{\tilde{r}}\right)$ and $A=0$, Eq. (23) (derived for SBD gravity) is converted into a non-linear homogeneous Lane-Emden type differential equation, which can be used to discuss polytropic filaments in BD gravity. Thus, numerical results obtained for $A=0$ and $n=0,1,2,3,5$ can indicate polytropic filaments in BD gravity. It is mentioned here that the polytropic 
models described by GR depend only upon the values of the polytropic index $n$. In the spherical case, the polytropic stars for $n<5$ have finite radii, while $n \geqslant 5$ provides infinite radii models [51]. In the cylindrical case, there exist models with finite radii for $0 \leqslant n<\infty$ [7]. Since we have derived filamentary polytopic models present in the universe from small scales to cosmic scales, the astrophysical length scale of our evaluated models are appropriate for both stellar size and cosmic large scales.

\section{Radial oscillations of polytropic filaments}

In this section, we discuss the stability as well as normal modes of radially oscillating filaments about their equilibrium configuration. For this purpose, we assume that initially the system is in complete hydrostatic equilibrium. Afterward, the stellar body starts oscillating (in the radial direction) and becomes perturbed $[15,51]$.

\subsection{Stability analysis}

In order to explore radially oscillating filamentary structures for different matter distributions in SBD gravity, we assume Lagrangian coordinates $(\mu, t)$, where $\mu$ represents the mass per unit length and $t$ stands for time. Let $r=r(\mu, t)$ be the distance of the mass from the center of the cylinder such that $\mu=\pi r^{2} \rho$ satisfies the following relations [51]:

$$
\begin{aligned}
\frac{\mathrm{d}}{\mathrm{d} \mu} & =\frac{1}{2 \pi r \rho} \frac{\mathrm{d}}{\mathrm{d} r}, \\
\frac{\mathrm{d} r}{\mathrm{~d} \mu} & =\frac{1}{2 \pi r \rho} .
\end{aligned}
$$

To study the stability criteria for an oscillating cylindrical filament, we consider a thin shell of mass element $\mathrm{d} \mu$ (per unit length). The shell faces a force $F_{g}$ per unit area from the gravitational field of

$F_{g}=g \rho \mathrm{d} r=-\frac{G_{(\text {eff })} \mu}{\pi r^{2}} \mathrm{~d} \mu-\frac{1}{2 \pi r} \frac{\Lambda_{\mathrm{BD}}}{3} \mathrm{~d} \mu$,

where $g=\frac{G_{(\mathrm{eff})} \mu}{r}+\frac{\Lambda_{\mathrm{BD}}}{3}$ represents gravitational potential due to the matter and scalar field. The pressure gradient forces exert a force $F_{P}$ per unit area of the shell given by

$F_{p}=-\frac{\mathrm{d} p}{\mathrm{~d} r} \mathrm{~d} r=-\frac{\mathrm{d} p}{\mathrm{~d} \mu} \mathrm{d} \mu$.

According to Eqs. (28), (29) and the $\mathrm{N}$ approximation (Newton's second law), the equation of motion for the shell becomes

$\frac{1}{2 \pi r} \frac{\mathrm{d}^{2} r}{\mathrm{~d} t^{2}}=-\frac{\mathrm{d} p}{\mathrm{~d} \mu}-\frac{G_{(\mathrm{eff})} \mu}{\pi r^{2}}-\frac{1}{2 \pi r} \frac{\Lambda_{\mathrm{BD}}}{3}$.
Let us perturb the following quantities adiabatically with a time dependent perturbation $e^{i \omega}$ ( $\omega$ indicates the frequency of the oscillations):

$$
\begin{aligned}
& r(\mu, t)=r_{0}(\mu)+r_{0}(\mu) \bar{r}(\mu) e^{i \omega}, \\
& \rho(\mu, t)=\rho_{0}(\mu)+\rho_{0}(\mu) \bar{\rho}(\mu) e^{i \omega}, \\
& p(\mu, t)=p_{0}(\mu)+p_{0}(\mu) \bar{p}(\mu) e^{i \omega},
\end{aligned}
$$

where the quantities with a zero subscript indicate unperturbed terms, while the terms with bar represent the perturbed ones. The perturbations are assumed to be very small such that $\frac{\bar{r}}{r_{0}}, \frac{\bar{\rho}}{\rho_{0}}$ and $\frac{\bar{p}}{p_{0}}$ are $<<<1$. After applying the perturbation scheme to Eq. (30), we obtain

$\frac{\mathrm{d} p_{0}}{\mathrm{~d} r_{0}}=g_{0} \rho_{0}$,

$\frac{\mathrm{d}}{\mathrm{d} \mu}\left(p_{0} \bar{p}\right)=\frac{\bar{r}}{2 \pi r_{0}}\left(2 g_{0}+r_{0} \omega^{2}\right)$,

where $g_{0}=\frac{G_{(\text {eff })} \mu}{r_{0}}+\frac{\Lambda_{\mathrm{BD}}}{3}$ and we have taken linear contributions of the perturbed terms. Equation (34) represents an unperturbed configuration, while Eq. (35) shows a perturbed form of the equation of motion of the shell.

Equations (26) and (35) provide the radial dependence of the perturbed equation of motion as follows:

$\frac{\mathrm{d} \bar{p}}{\mathrm{~d} r_{0}}=\frac{\rho_{0}}{p_{0}}\left[r_{0} \bar{r} \omega^{2}+g_{0}(2 \bar{r}+\bar{p})\right]$,

while the perturbed configuration of Eq. (27) becomes

$r_{0} \frac{\mathrm{d} \bar{r}}{\mathrm{~d} r_{0}}=-2 \bar{r}-\bar{\rho}$.

Since the system is perturbed adiabatically, the perturbed density and pressure are related by

$\bar{p}=\Gamma_{(\text {ad })} \bar{\rho}$.

Here $\Gamma_{(\mathrm{ad})}$ is a constant term representing the adiabatic exponent. Using Eqs. (37) and (38) in (36), we obtain

$$
\begin{aligned}
& \bar{r}^{\prime \prime}+\left[\frac{3}{r_{0}}-\frac{\rho_{0} g_{0}}{p_{0}}\right] \bar{r}^{\prime} \\
& +\frac{\rho_{0}}{\Gamma_{\mathrm{ad}} P_{0}}\left(\omega^{2}+2\left[1-\Gamma_{(\mathrm{ad})}\right] \frac{g_{0}}{r_{0}}\right) \bar{r}=0,
\end{aligned}
$$

where a prime indicates the derivative with respect to $r_{0}$. This is a second order differential equation which represents the relative amplitude $\left(\bar{r}\left(r_{0}\right)\right)$ as a function of depth for a radial adiabatic oscillation of frequency $\omega$. Equation (39) can be converted into a standard Sturm-Liouville (SL) equation if it is multiplied by a factor $p_{0} r_{0}^{3}$ as follows:

$$
\left(r_{0}^{3} p_{0} \bar{r}^{\prime}\right)^{\prime}+\frac{r_{0}^{3} \rho_{0}}{\Gamma_{(\mathrm{ad})}}\left(\omega^{2}+2\left[1-\Gamma_{(\mathrm{ad})}\right] \frac{g_{0}}{r_{0}}\right) \bar{r}=0 .
$$

According to SL theory [51], the term $\omega^{2}$ behaves as an eigenvalue for the SL problem and there exist an infinite number 
of eigenvalues $\left(\omega_{n}\right)$ satisfying the property $\omega_{n+1}^{2}>\omega_{n}^{2}$. For each eigenvalue, there is a corresponding eigenfunction $\bar{r}_{n}$, which represents an amplitude of oscillations with a number of $n$ nodes in the range $0<r_{0}<R_{0}$. The lowest-order eigenfunction $\bar{r}_{0}$ shows no node and is known as the fundamental amplitude.

It is mentioned here that stability, instability, and marginal stability of any oscillating model depend upon the behavior of its frequency. If the frequency of the model is real, the perturbation is purely oscillatory with constant amplitude providing a dynamically stable equilibrium, while imaginary values of the frequency lead to periodic oscillations in which the amplitude increases exponentially in time and the system becomes dynamically unstable. If the frequency tends to zero, the system becomes marginally stable (neither stable nor unstable), i.e., the model will expand and contract with the homologous property [51].

In order to evaluate the stability criteria for a radial perturbation, we solve Eq. (40) for the fundamental mode with boundary condition $p_{0}\left(R_{0}\right)=0$. We insert the eigenfunction $\bar{r}_{0}$ in the SL equation and integrate over $0<r_{0}<R_{0}$; it follows that

$$
\begin{aligned}
& {\left[r_{0}^{3} p_{0} \bar{r}_{0}^{\prime}\right]_{0}^{R_{0}}+\frac{\omega_{0}^{2}}{\Gamma_{(\mathrm{ad})}} \int_{0}^{R_{0}} r_{0}^{3} \rho_{0} \bar{r}_{0} \mathrm{~d} r_{0}} \\
& +\frac{2-2 \Gamma_{(\mathrm{ad})}}{\Gamma_{(\mathrm{ad})}} \int_{0}^{R_{0}} r_{0}^{2} \rho_{0} g_{0} \bar{r}_{0} \mathrm{~d} r_{0}=0 .
\end{aligned}
$$

Since $\bar{r}_{0}^{\prime}$ is finite everywhere, the first term in the above equation vanishes, yielding

$$
\omega_{0}^{2}=2\left(\Gamma_{(\mathrm{ad})}-1\right) \frac{\int_{0}^{R_{0}} r_{0}^{2} \rho_{0} g_{0} \bar{r} \mathrm{~d} r_{0}}{\int_{0}^{R_{0}} r_{0}^{3} \rho_{0} \bar{r}_{0} \mathrm{~d} r_{0}} .
$$

This equation provides the fundamental frequency of the filaments, which leads to the stability criterion of the system. If $\omega_{0}^{2}>0$ then $\omega_{n}^{2}>\omega_{0}^{2}>0$ for all nodes $n>0$ and the frequency $\left( \pm \omega_{n}\right)$ becomes real for all values of $n$, leading to dynamically stable filaments. Similarly, $\omega_{0}^{2}<0 \Rightarrow \omega_{n}^{2}<0$ for a finite number of nodes and the frequency becomes imaginary, providing unstable configurations. Otherwise $\omega_{0}^{2}=0$ gives a marginally stable configuration.

Since the fundamental amplitude represents no node, it has the same sign all over the cylindrical filament and hence the two integrals in Eq. (42) have the same sign. Thus, the stability criterion depends upon sign $\omega_{0}^{2}=\operatorname{sign} 2\left(\Gamma_{(\mathrm{ad})}-1\right)$. If

$\Gamma_{(\text {ad })}>1$

$\omega_{0}^{2}>0$, and the corresponding model becomes stable. For

$\Gamma_{(\mathrm{ad})}=1$, $\omega_{n}^{2}=0$, and the system is marginally stable. When

$\Gamma_{(\mathrm{ad})}<1$,

$\omega_{0}^{2}<0$, and the system becomes dynamically unstable.

Thus, the criterion of stability for the filamentary structures in the $\mathrm{N}$ limit of SBD gravity depends only upon the stiffness of the fluid $\left(\Gamma_{(\mathrm{ad})}\right)$. The stability conditions are independent from the behaviors of dynamical variables related to the metric, the coupling constant $\left(\omega_{\mathrm{BD}}\right)$, the scalar field, and the potential of the scalar field.

It is mentioned here that in GR, the stability of spherical as well as cylindrical polytropic models depends only upon the stiffness parameter. In the spherical case, $\Gamma_{(\text {ad })}<\frac{4}{3}$ leads to an unstable model, while cylindrically symmetric filaments remain stable for $\Gamma_{(\mathrm{ad})}>1[15,50]$. In the case of BD gravity, the spherically symmetric polytropic model remains unstable for $\Gamma_{(\mathrm{ad})}>\frac{4}{3}$ [52], while cylindrically symmetric polytropic models are not discussed. Thus, our obtained results are consistent with GR in the $\mathrm{N}$ regime.

\subsection{Normal modes of radial oscillations}

The behavior of normal modes for polytropic filaments can be described by using polytropic quantities in the SL problem. We use Eqs. (15), (20), (22) in (40), and it follows that

$$
\begin{aligned}
& \frac{\mathrm{d} \bar{r}^{2}}{\mathrm{~d} s^{2}}+\left[\frac{3}{s}+\frac{n+1}{\beta} \frac{\mathrm{d} \beta}{\mathrm{d} s}-\frac{\Lambda_{\mathrm{BD}}}{3}\right] \frac{\mathrm{d} \bar{r}}{\mathrm{~d} s}-\left[b^{2} \frac{n+1}{\Gamma_{(\mathrm{ad})} h_{00(c)}} \omega^{2}\right. \\
& \left.\quad+2\left(1-\Gamma_{(\mathrm{ad})}\right) \frac{n+1}{\Gamma_{(\mathrm{ad})} s} \frac{\mathrm{d} \beta}{\mathrm{d} s}\right] \frac{\bar{r}}{\beta}=0
\end{aligned}
$$

In order to solve this equation for $\bar{r}$, the required boundary conditions are as follows: at the center of cylinder, we have $\left(\frac{\mathrm{d} \bar{r}}{\mathrm{~d} s}\right)_{s=0}=0$, at the boundary of polytrope $s=S$, we have $\frac{p_{0}}{\rho_{0}}<<<1$, and the values of $\bar{r}$ remain finite. Thus, Eqs. (36) and (37) yield

$\left[\omega^{2} b S+g_{0}\left(2-2 \Gamma_{(\mathrm{ad})}\right)\right] \bar{r}(S)-g_{0} \Gamma_{(\mathrm{ad})}\left(\frac{\mathrm{d} \bar{r}}{\mathrm{~d} s}\right)_{s=S}=0$.

Equation (46) along with boundary conditions provides different patterns of normal modes of oscillating polytropic filaments in SBD theory. It is mentioned here that the behavior of these normal modes depends upon the frequency, the central potential, the polytropic index, the adiabatic exponent, and the gravitational potential term due to the massive scalar field $\left(g_{0}\right)$, i.e., for different values of these parameters, we have different modes of radial oscillations of polytropic filaments.

\section{Final remarks}

This paper investigates cylindrically symmetric filamentary structures in the $\mathrm{N}$ limit of SBD gravity. We have formulated a 
generalized form of the Lane-Emden equation in $\mathrm{N}$ approximations and obtained polytropic filament models analytically for $n=0,1$ as well as numerically for $n=0,1,3,5$. We have found that the behavior of these models depend upon the values of polytropic index as well as the cosmological constant term $A$ (due to the scalar field). For $n=0$, the models have finite radii for $A \lesssim 1 / 2$, otherwise they have infinite radii. For $n=1,3,5$, we have approximated that $A \lesssim 0$ represents finite radii polytropic filaments while $A>0$ gives infinite radii models. We have also found that within $m_{0}<<\frac{1}{\tilde{r}}$ and $A=0$, the models defined for $A=0$ and $n=0,1,2,3,5$ are same for BD gravity.

In order to study fragmentation of the filamentary structures, we have investigated the stability of radial oscillations of polytropic filaments. It is found that the stability criterion of cylindrical filaments in SBD gravity depends only upon the adiabatic index $\left(\Gamma_{\mathrm{ad}}\right)$. Generally in the weak field approximation of GR and BD gravity, the stability criterion depends upon the adiabatic index as well as on the dynamical variables related to matter and scalar field distributions. But in the polytropic case, the adiabatic index is responsible for the stability of the function. Finally, we have discussed possible normal modes of radial oscillations of polytropic filaments. It turns out that different values of parameters lead to different modes of radially oscillating filaments. It is found that the weak field approximation of SBD gravity is consistent with observations for all arbitrary values $\omega_{\mathrm{BD}}$ and hence our obtained results are valid for all arbitrary values of $\omega_{\mathrm{BD}}$. It is interesting to mention here that our results provide a generalized form of cylindrical filament polytropic models of GR theory.

Open Access This article is distributed under the terms of the Creative Commons Attribution 4.0 International License (http://creativecomm ons.org/licenses/by/4.0/), which permits unrestricted use, distribution, and reproduction in any medium, provided you give appropriate credit to the original author(s) and the source, provide a link to the Creative Commons license, and indicate if changes were made. Funded by SCOAP ${ }^{3}$.

\section{References}

1. A. Jenkins et al., Astrophys. J. 499, 20 (1998)

2. D.A. Turnshek et al., Astrophys. J. 609, L53 (2004)

3. J.M. Colberg et al., Mon. Not. Roy. Astron. Soc. 359, 272 (2005)

4. P.A.R. Ade et al., Astron. Astrophys. 550, A134 (2013)
5. P.C. Myers, Astrophys. J. 764, 140 (2013)

6. S. Chandrasekhar, E. Fermi, Astrophys. J. 118, 116 (1953)

7. J.S. Stodolkiewicz, Astron. Astrophys. 13, 30 (1963)

8. J. Ostriker, Astrophys. J. 140, 1056 (1964)

9. J. Ostriker, Astrophys. J. 140, 1029 (1964)

10. M. Milgrom, Astrophys. J. 478, 7 (1997)

11. A. Kneb et al., Mon. Not. Roy. Astron. Soc. 345, 1285 (2003)

12. L. Gao, T. Thenus, Sci. 317, 1527 (2007)

13. S. Bessho, T. Tsuribe, Publ. Astron. Soc. Jpn. 64, 104 (2012)

14. J. Freundlich et al., Astron. Astrophys. 564, A7 (2014)

15. P.C. Breysse et al., Mon. Not. Roy. Astron. Soc. 437, 2675 (2014)

16. M. Mohseni, Phys. Lett. B 682, 89 (2009)

17. A.D. Felice, S. Tsujikawa, Phys. Rev. D 80, 063516 (2009)

18. T. Harko et al., Phys. Rev. D 84, 024020 (2011)

19. E. Papantonopoulos, Modifications of Einstein's Theory of Gravity at Large Distance (Springer, 2014)

20. P. Chang, L. Hui, Astrophys. J. 732, 25 (2011)

21. B. Jain, J. VanderPlas, J. Cosmol. Astropart. Phys. 10, 032 (2011)

22. A.C. Davis et al., Phys. Rev. D 85, 123006 (2012)

23. M. Sharif, Z. Yousaf, Phys. Rev. D 88, 024020 (2013)

24. M. Sharif, R. Manzoor, Mod. Phys. Lett. A 29, 1450192 (2014)

25. M. Sharif, R. Manzoor, Astrophys. Space Sci. 354, 497 (2014)

26. P.A.M. Dirac, Proc. R. Soc. Lond. A 165, 199 (1938)

27. C.H. Brans, R.H. Dicke, Phys. Rev. 124, 925 (1961)

28. E.J. Weinberg, Phys. Rev. D 40, 3950 (1989)

29. R.D. Reasenberg et al., Astrophys. J. 234, L219 (1979)

30. B.I.L. Bertotti, P. Tortora, Nature 425, 374 (2003)

31. A.D. Felice et al., Phys. Rev. D 74, 103005 (2006)

32. N. Banerjee, D. Pavon, Phys. Rev. D 63, 043504 (2001)

33. C. Santos, R. Gregory, Annals Phys. 258, 111 (1997)

34. Y. Bisabr, Gen. Relativ. Gravity 44, 427 (2012)

35. V. Faraoni, Phys. Rev. D 62, 023504 (2000)

36. M.K. Mak, T. Harko, Europhys. Lett. 60, 155 (2002)

37. Y. Bisaby, Astrophys. Space Sci. 339, 1 (2012)

38. M. Sharif, S. Waheed, Eur. Phys. J. C 72, 1876 (2012)

39. M. Sharif, S. Waheed, J. Phys. Soc. Jpn. 81, 114901 (2012)

40. O. Hrycyna et al., Phys. Rev. D 90, 124040 (2014)

41. M. Sharif, R. Manzoor, Astrophys. Space Sci. 359, 17 (2015)

42. M. Sharif, R. Manzoor, Phys. Rev. D 91, 024018 (2015)

43. M. Sharif, R. Manzoor, Gen. Relativ. Gravity 47, 98 (2015)

44. M. Sharif, R. Manzoor, Eur. Phys. J. Plus 131, 16064 (2016)

45. M. Sharif, R. Manzoor, Stability of Gaseous Masses for Radial Oscillations in Massive Gravity (submitted for publication)

46. G.J. Olmo, Phys. Rev. D 72, 083505 (2005)

47. R.V. Wagoner, Phys. Rev. D 1, 3209 (1970)

48. P.J. Steinhardt, C.M. Will, Phys. Rev. D 52, 628 (1995)

49. L. Perivolaropoulos, Phys. Rev. D 67, 123516 (2003)

50. S. Chandrasekhar, An Introduction to the Study of Stellar Structure (Dover editions, 1967)

51. R. Kippenhahn, A. Weigert, Stellar Structure and Evolution (Springer, 1990)

52. Y. Nutku, Astrophys. J. 155, 999 (1969) 